\title{
Low-Temperature Phases in Two-Orbital Hubbard Model Realized with Ultracold Atoms in Optical Lattices
}

\author{
A. Sotnikov ${ }^{a}$, Y. ZAMBrano ${ }^{b}$ And A. CICHY ${ }^{b, c, *}$ \\ ${ }^{a}$ Akhiezer Institute for Theoretical Physics, NSC KIPT, \\ Akademichna 1, 61108 Kharkiv, Ukraine \\ ${ }^{b}$ Faculty of Physics, Adam Mickiewicz University, \\ Uniwersytetu Poznańskiego 2, PL-61-614 Poznań, Poland \\ ${ }^{c}$ Institut für Physik, Johannes Gutenberg-Universität Mainz, \\ Staudingerweg 9, D-55099 Mainz, Germany \\ Doi: $10.12693 /$ APhysPolA.138.669 \\ *e-mail: agnieszkakujawa2311@gmail.com

\begin{abstract}
We study ultracold quantum gases of alkaline-earth-like atoms loaded into three-dimensional statedependent optical lattice. In particular, we focus on the fermionic ytterbium-173 atoms due to their unique properties, in particular, low-lying metastable excited $e$ electronic state, decoupling of the nuclear spin from the electronic degrees of freedom and different AC-polarizabilities of the ground $g$ and $e$ states. This allows to realize the two-band Hubbard model and study its unique critical properties. The theoretical analysis is performed in the region of applicability of the tight-binding approximation at different lattice depths and different fillings by atoms in the $g$ - and $e$-orbital states. By means of dynamical mean-field theory, we analyze stability regions of the emerging ordered phases.
\end{abstract}

topics: Ultracold atoms, optical lattices, two-band Hubbard model, ytterbium, ferromagnetism, orbital ordering

\section{Introduction}

In recent years, the realization of universal quantum simulators [1] by means of ultracold gases in optical lattices becomes more and more consequential [2]. Among the inspiring examples, observations of long-range antiferromagnetic (AFM) correlations of ultracold ${ }^{6} \mathrm{Li}$ atoms [3] provide a fresh outlook to the scientific community. Now, debates are centered not only around additional cooling mechanisms but also around particular systems and further physical regimes, where these simulators can be successfully applied. In this respect, alkaline-earthlike (AEL) atoms, in particular, fermionic isotopes of $\mathrm{Yb}$ and $\mathrm{Sr}$, demonstrate appropriate capabilities $[4,5]$ to account for orbital degrees of freedom vital in strongly-correlated solid-state materials. The extension to the two-orbital Hubbard model already uncovers layers of captivating physical phenomena, ranging from the Kondo effect and orbitalselective Mott transitions to ferromagnetism and colossal magnetoresistance.

In this paper, we study orbital ordering in the two-band Hubbard model (2BHM) including Hund's exchange coupling term which can be realized in ultracold four-component mixtures of AEL atoms in optical lattices. We employ dynamical mean-field theory (DMFT) to estimate critical temperatures and stability regions at various lattice depths. The paper is organized as follows: we shortly introduce the model and the method (Sec. 2). In Sect. 3, we present and discuss numerical results and finally, we summarize our results in Sect. 4.

\section{Model and method}

We describe the system with a Hamiltonian:

$$
\begin{aligned}
\mathcal{H} & =\sum_{\langle i, j\rangle \gamma \sigma} t_{\gamma}\left(c_{i \gamma \sigma}^{\dagger} c_{j \gamma \sigma}+\text { h.c. }\right)-\sum_{i \gamma} \mu_{\gamma} n_{i \gamma} \\
& +\sum_{i \gamma} U_{\gamma} \sum_{\sigma<\sigma^{\prime}} n_{i \gamma \sigma} n_{i \gamma \sigma^{\prime}}+V \sum_{\substack{i \sigma<\sigma^{\prime}, \gamma \neq \gamma^{\prime}}} n_{i \gamma \sigma} n_{i \gamma^{\prime} \sigma^{\prime}} \\
& +\left(V-V_{\mathrm{ex}}\right) \sum_{\substack{i \sigma, \gamma<\gamma^{\prime}}} n_{i \gamma \sigma} n_{i \gamma^{\prime} \sigma} \\
& +V_{\mathrm{ex}} \sum_{\substack{i, \sigma<\sigma^{\prime}, \gamma \neq \gamma^{\prime}}} c_{i \gamma \sigma}^{\dagger} c_{i \gamma^{\prime} \sigma^{\prime}}^{\dagger} c_{i \gamma \sigma^{\prime}} c_{i \gamma^{\prime} \sigma}
\end{aligned}
$$

where $\gamma=\{g, e\}$ denotes the electronic state representing the orbital, $\sigma=\{-I, \ldots, I\}$ is one of the $\mathcal{N}=2 I+1$ nuclear Zeeman spin states (we restrict below to $\mathcal{N}=2), c_{i \gamma \sigma}^{\dagger}\left(c_{i \gamma \sigma}\right)$ corresponds to the creation (annihilation) operator of an atom at the site $i$ in the internal state $|\gamma \sigma\rangle, t_{\gamma}$ is 
the hopping amplitude, $\mu_{\gamma}$ is the chemical potential, $n_{i \gamma \sigma}=c_{i \gamma \sigma}^{\dagger} c_{i \gamma \sigma}$ and $n_{i \alpha}=\sum_{\sigma} n_{i \gamma \sigma}$. The intraorbital interaction is denoted as $U_{\gamma}$, while $V$ and $V_{e x}$ describe the on-site interorbital direct and exchange interaction terms, respectively.

We perform a numerical analysis within DMFT formalism. The main idea of the DMFT approach is to map the quantum lattice problem with many degrees of freedom onto a single site - the "impurity site" — coupled self-consistently to a noninteracting bath. In contrast to the static meanfield approach, the local dynamics of a DMFT effective problem is treated exactly. Hence, although it is not an exact method, it provides both qualitatively and quantitatively reasonable estimates which can be compared to experimental measurements in quasi-2D and 3D systems (in this paper we focus on the 3D case with the lattice coordination number $z=6$ ). In the theoretical analysis, the Hamiltonian (1) is mapped onto the Anderson impurity model. The most challenging part of the procedure is the impurity problem which is treated by the exact diagonalization solver preserving the $\mathrm{SU}(2)$ symmetry in the spin space [6]. The maximal number of effective bath orbitals is limited by $n_{s}=4$ per each orbital and spin component.

The DMFT self-consistency condition in terms of Green's functions $G_{\gamma \sigma}$ for homogeneous, including ferromagnetic (FM) and normal (N), phases in the presence of the magnetic field $h$ takes the form:

$$
G_{\gamma \sigma}\left(\mathrm{i} \omega_{n}\right)=\int \frac{D_{\gamma}(\epsilon) \mathrm{d} \epsilon}{\mathrm{i} \omega_{n}+\mu+h \sigma-\epsilon-\Sigma_{\gamma \sigma}\left(\mathrm{i} \omega_{n}\right)},
$$

where $D_{\gamma}(\epsilon)$ is the density of states of noninteracting atoms in the orbital state $\gamma$, the fermionic Matsubara frequency is denoted as $\omega_{n}=(2 n+1) \pi T$ and $\Sigma_{\gamma \sigma}\left(\mathrm{i} \omega_{n}\right)$ is the self-energy obtained by solving the impurity problem. The magnetization as a function of the external field is given by:

$$
m(h)=\frac{1}{\beta} \sum_{\gamma, n} \mathrm{e}^{\mathrm{i} \omega_{n} 0^{+}}\left(G_{\gamma \uparrow}\left(\mathrm{i} \omega_{n}\right)-G_{\gamma \downarrow}\left(\mathrm{i} \omega_{n}\right)\right) .
$$

The ferromagnetic phase is signaled by a nonzero spontaneous magnetization $m_{0}$, where $m_{0}=\lim _{h \rightarrow 0} m(h) \neq 0$. The fits for magnetic susceptibility $\chi$ can be written as:

$$
m=m_{0}+\chi h,
$$

where $m$ is the average magnetization, $m_{0}$ is the residual magnetization and $\chi$ is determined from the slope of the magnetization line. The magnetic susceptibility diverges at the critical temperature $T_{c}$. Thus, the temperatures below which the FM phase is stable can be determined by the Curie-Weiss fit:

$$
\chi=\frac{c}{T-T_{c}},
$$

where $c$ is the fitting parameter.
To detect two-sublattice ordering, e.g., AFM or antiferroorbital AFO long-range correlations, the DMFT self-consistency conditions are extended to the following form:

$$
G_{\gamma \sigma \alpha}\left(i \omega_{n}\right)=\int \frac{\zeta_{\gamma \sigma \bar{\alpha}} D_{\gamma}(\epsilon) \mathrm{d} \epsilon}{\zeta_{\gamma \sigma A} \zeta_{\gamma \sigma B}-\epsilon^{2}}
$$

with $\zeta_{\gamma \sigma \alpha} \equiv \mathrm{i} \omega_{n}+\mu_{\gamma}-\Sigma_{\gamma \sigma \alpha}\left(\mathrm{i} \omega_{n}\right)$ and the sublattice indices $\alpha=A, B$ and their opposites $\bar{\alpha}=B, A$.

\section{Numerical results and discussion}

For simplicity, we choose the state-dependent optical lattice acting on ultracold ${ }^{173} \mathrm{Yb}$ atoms with the same amplitude in all three spatial directions (cubic lattice geometry). Only the selected results will be presented below, while findings of an extended study, in particular, with more experimentally-relevant quasi-2D lattice geometry are given in Ref. [7]. The Hamiltonian (1) implies a single-band approximation. Hence, we consider the case of a sufficiently strong lattice potential in all spatial directions $s \geq 3 E_{r}$, where $E_{r}=\hbar^{2} k^{2} / 2 M$ is the recoil energy and $k$ is the wave number determined by the wavelength $\lambda$ of the laser forming the isotropic cubic optical lattice.

To provide theoretical estimates of the Hubbard parameters entering the model (1), we employ the band-structure analysis [8] with the knowledge of the scattering lengths of atoms in different states, the variable laser intensity and the fixed laser wavelength $\lambda=670 \mathrm{~nm}$ with the corresponding ratio of differential polarizabilities of atoms in $g$ and $e$ states $\alpha_{e}(\omega) / \alpha_{g}(\omega) \approx 3.3$ [9]. The intraorbital interaction amplitudes are determined as $U_{\gamma}=g_{\gamma \gamma} \int \mathrm{d}^{3} r w_{\gamma}^{4}(\boldsymbol{r})$, while $V=\left(U_{e g}^{+}+U_{e g}^{-}\right) / 2$ and $V_{\mathrm{ex}}=\left(U_{e g}^{+}-U_{e g}^{-}\right) / 2$. Here $g_{\gamma \gamma^{\prime}}=4 \pi \hbar^{2} a_{\gamma \gamma^{\prime}} / M$, where $a_{\gamma \gamma^{\prime}}$ is the scattering length of two atoms on orbitals $\gamma$ and $\gamma^{\prime}, M$ is the atomic mass, $w_{\gamma}(\boldsymbol{r})$ is the Wannier function on the orbital $\gamma$ and $U_{e g}^{ \pm}=g_{\text {eg }}^{ \pm} \int \mathrm{d}^{3} r w_{e}^{2}(\boldsymbol{r}) w_{g}^{2}(\boldsymbol{r})$. In the case under study, $a_{\text {lat }} \simeq 6 \times 10^{3} a_{0}$ is the lattice constant with $a_{0}$ being the Bohr radius and the scattering lengths are $a_{g g}=199 a_{0}, a_{e e}=306 a_{0}$, $a_{e g}^{-}=220 a_{0}$ and $a_{e g}^{+} \simeq 1900 a_{0}[10,11]$.

The dependencies of Hubbard parameters on the lattice depth $s$ are shown in Fig. 1. The ratio $t_{e} / t_{g}$ can be tuned by adjusting the depth of the optical lattice or by changing its wavelength. As it can be seen from Fig. 1, the hierarchy of the on-site interactions in ultracold AEL atoms in state-dependent optical lattices is different from the Coulomb-type parametrization employed in strongly-correlated electron systems. Namely, for the latter, the relation $V=U-2 V_{\text {ex }}$ is valid for all electron-electron interactions which are rotationally invariant in real space. However, the on-site interaction amplitudes of ${ }^{173} \mathrm{Yb}$ atoms in the system under study demonstrate the hierarchy of the type $U_{g g}<U_{e e}<V_{\mathrm{ex}}<V$ due to the relatively large scattering length of the orbitally-symmetric state.

Next, we focus on one particular case of the total filling $n=1.5$, where, depending on the ratio $n_{g} / n_{e}$, 


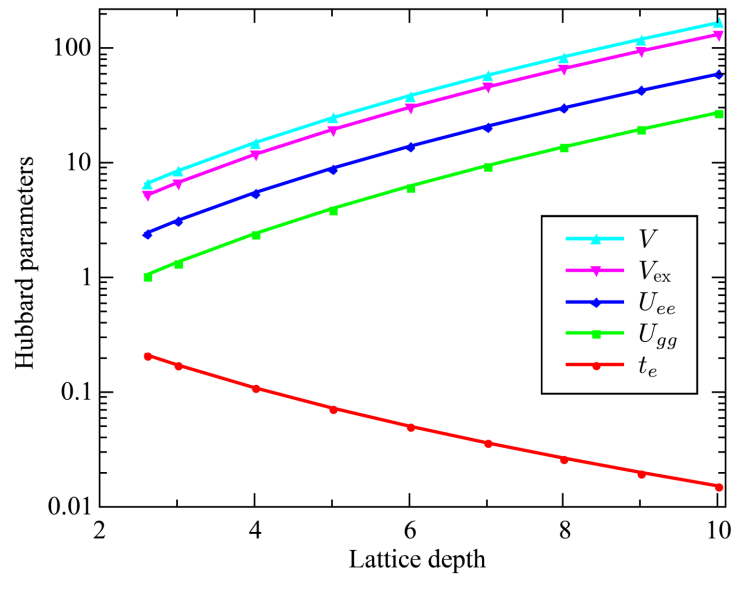

Fig. 1. Hubbard parameters in units of the hopping amplitude $t_{g}$ obtained from the band-structure calculation as functions of the lattice depth $s$ in units of $E_{r}$ at the fixed laser wavelength $\lambda=670 \mathrm{~nm}$.
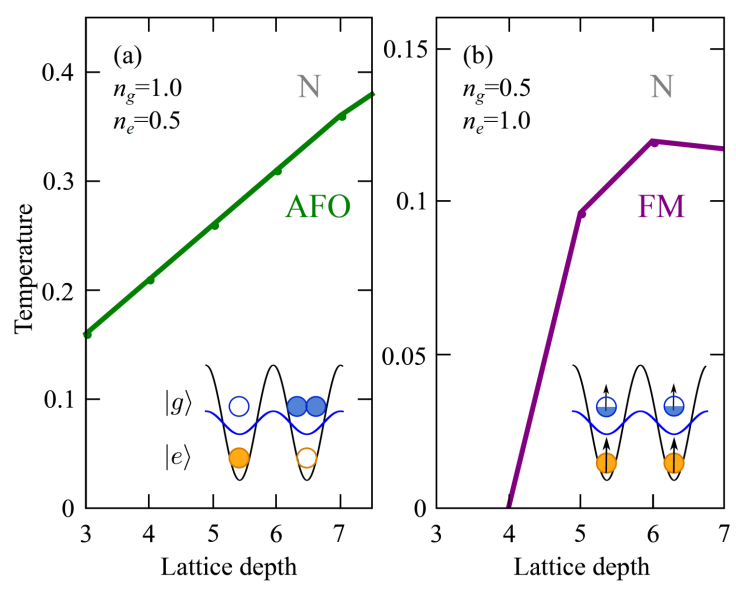

Fig. 2. Critical temperatures in units of $t_{g}$ as functions of the lattice depth $s$ in units of $E_{r}$ in the regions of the AFO (a) and FM (b) instabilities. The insets schematically indicate the average filling of two adjacent lattice sites by orbital components in the corresponding phases.

a formation of the AFO or FM phases at sufficiently low temperatures is observed (see Fig. 2). The AFO long-range ordering is characterized by two spatially-alternating sublattices: one of them consists mostly of sites occupied by a single atom in the $e$ state and the other is filled by pairs of $g$ atoms. Hence, the above-mentioned filling with $n_{g} \approx 1$ and $n_{e} \approx 0.5$ can be viewed as the most appropriate for the detection of the AFO correlations.

In Fig. 2a, we explore the influence of lattice depth on the AFO ordering stability with respect to thermal fluctuations. As it can be observed, the critical temperature in units of $t_{g}$ for the AFO phase increases linearly with the increase of the lattice depth. For large lattice potentials, the hopping becomes more suppressed and interactions become

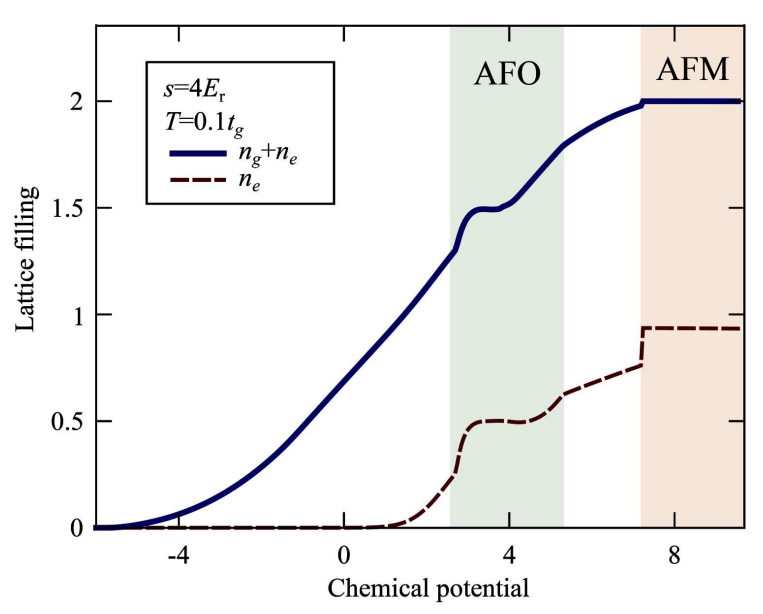

Fig. 3. Density profiles as functions of the average chemical potential $\mu=\left(\mu_{g}+\mu_{e}\right) / 2$ in units of $t_{g}$. The highlighted areas indicate the stability regions of the AFO and AFM phases with respect to changes in the density of atoms.

stronger. Moreover, the Mott-insulator plateau is revealed at $n=1.5$ which is clearly visible in the dependence of the total filling on the chemical potential (see Fig. 3). Hence, in this regime, the AFO order becomes energetically favorable due to a spatial separation of $g$ atoms which have the smallest interaction amplitude. It is worth mentioning that, according to the DMFT analysis, the AFO also remains stable substantially away from the plateau with $n_{g}=1$ and $n_{e}=0.5$ (see the indicated region in Fig. 3), while the maximum for the AFO critical temperature is observed at $n=1.5$.

From the point of view of experiments dealing with ultracold atoms in optical lattices, the entropy is an important thermodynamical quantity. The calculations involving the local density approximation in addition to DMFT $\left(\mu(r)=\mu_{0}-V_{\mathrm{tr}} r^{2}\right.$, where $V_{\mathrm{tr}}$ is the trapping potential, $r$ is the distance from the trap center and $\mu_{0}$ is the chemical potential in the center of the trap) provide us with expectation values of the local density $n$ of atoms at different values of the chemical potential. Given these quantities at different temperatures, the entropy per site can be obtained from the Maxwell relation,

$$
S\left(\mu_{0}, T\right)=\int_{-\infty}^{\mu_{0}}\left(\frac{\partial n}{\partial T}\right) \mathrm{d} \mu .
$$

Now, using (7) at $s=4$ and temperatures close to $T_{c}=0.21 t_{g}$ (see Fig. $\left.2 \mathrm{a}\right), n_{g}^{(0)}=1$ and $n_{e}^{(0)}=0.5$ in the trap center (see, e.g. Fig. 3 at $\mu \approx 3.5$ ), we estimate the critical entropy per particle, i.e., $S / N \approx 0.885 k_{\mathrm{B}}$. This value is currently slightly below the lower bound for entropies per particle accessed in experiments with ${ }^{173} \mathrm{Yb}$. However, the AFO phase has advantages in comparison to the AFM phase in the Hubbard model, both in the detection techniques and the critical entropy values. 
Another interesting aspect of the analysis is the FM phase stability. It is well known that in solid state systems, Hund's coupling plays a key role in FM ordering and can be understood as a source of ferromagnetism in transition-metal oxides [12-14]. As it was shown in [13] for the limit of the Isingtype Hund's coupling, ferromagnetism is stabilized by the exchange interaction $V_{\text {ex }}$. However, according to the extended DMFT analysis [14], the more symmetric parametrization of interactions in multiorbital Hubbard model can significantly affect critical properties. In particular, a strong suppression of FM is observed when full spin-rotational symmetry of the Coulomb interaction in the $2 \mathrm{BHM}$ is taken into account.

In this study, we observe that for $\mathrm{SU}(2)$ symmetric interactions and their systemspecific AEL-atom-type parametrization, i.e., $U_{g g}<U_{e e}<V_{\text {ex }}<V$, the FM correlations can be stabilized in a wide region of interactions and lattice depths, see Fig. 2b. We also determine a critical value of the lattice depth $\left(s \approx 4 E_{r}\right)$ below which the FM correlations become suppressed at $T>0$, while the maximum for the Curie temperature in units of $t_{g}$ is observed at $s \approx 6 E_{\mathrm{r}}$. At stronger couplings, the critical temperature decreases slowly with the increase of the lattice depth.

\section{Summary}

In this paper, we have theoretically studied lowtemperature properties of gases consisting of AEL atoms loaded into state-dependent optical lattices, recently realized in experiments. These systems are currently of high relevance due to appropriate capabilities to realize, in particular, the twoband Hubbard model, the Kugel-Khomskii model, the Kondo lattice model and $\mathrm{SU}(\mathcal{N})$-symmetric magnetic systems.

We have analyzed magnetic and orbital properties of the two-orbital Hubbard model on the simple cubic lattice. It is shown that for the interaction parameters related to the experiments with fermionic ${ }^{173} \mathrm{Yb}$ atoms, orbital and magnetic ordering can emerge at specific lattice fillings. We have obtained temperature vs. lattice depth phase diagrams at $n=1.5$ and analyzed the range of occurrence of the AFO and the FM long-range correlations. We have estimated the critical entropy per particle below which the AFO ordering becomes stable and concluded that, although the value is low to detect this phase in the current experimental setups with ultracold ytterbium atoms, the AFO phase has advantages in comparison to the AFM phase observed in the single-band Hubbard model. We have indicated differences between the solid-state and the AEL-atom parametrizations of interactions in the 2BHM and shown that, in the latter case, ferromagnetism can be stabilized for the $\mathrm{SU}(2)$-symmetric parametrization of local interactions.

\section{Acknowledgments}

We thank Luis Riegger for providing experimental data and Nelson Darkwah Oppong for fruitful discussions. A.S. acknowledges funding by the Ministry of Education and Science of Ukraine, Research Grant No. 0120U102252. Y.Z. and A.C. acknowledge funding of this work by the National Science Centre (NCN, Poland) under Grant No. UMO2017/24/C/ST3/00357. Access to computing and storage facilities provided by the Poznan Supercomputing and Networking Center (EAGLE cluster) is greatly appreciated.

\section{References}

[1] R.P. Feynman, Int. J. Theor. Phys. 21, 467 (1982).

[2] K. Sandholzer, Y. Murakami, F. Görg, J. Minguzzi, M. Messer, R. Desbuquois, M. Eckstein, P. Werner, T. Esslinger, Phys. Rev. Lett. 123, 193602 (2019).

[3] A. Mazurenko, C.S. Chiu, G. Ji, M.F. Parsons, M. Kanász-Nagy, R. Schmidt, F. Grusdt, E. Demler, D. Greif, M. Greiner, Nature 545, 462 (2017).

[4] M. Foss-Feig, M. Hermele, A.M. Rey, Phys. Rev. A 81, 051603 (2010).

[5] A.V. Gorshkov, M. Hermele, V. Gurarie, C. Xu, P.S. Julienne, J. Ye, P. Zoller, E. Demler, M.D. Lukin, A.M. Rey, Nature Physics 6, 289 (2010).

[6] A. Golubeva, A. Sotnikov, A. Cichy, J. Kuneš, W. Hofstetter, Phys. Rev. B 95, 125108 (2017).

[7] A. Sotnikov, N.D. Oppong, Y. Zambrano, A. Cichy, Phys. Rev. Res. 2, 023188 (2020).

[8] I. Bloch, J. Dalibard, W. Zwerger, Rev. Mod. Phys. 80, 885 (2008).

[9] L. Riegger, N. Darkwah Oppong, M. Höfer, D.R. Fernandes, I. Bloch, S. Fölling, Phys. Rev. Lett. 120, 143601 (2018).

[10] F. Scazza, C. Hofrichter, M. Höfer, P.C. De Groot, I. Bloch, S. Fölling, Nat. Phys. 10, 779 (2014).

[11] M. Höfer, L. Riegger, F. Scazza et al., Phys. Rev. Lett. 115, 265302 (2015).

[12] K. Held, D. Vollhardt, Eur. Phys. J. B 5, 473 (1998).

[13] A. Cichy, A. Sotnikov, Phys. Rev. A 93, 053624 (2016).

[14] A. Sotnikov, A. Cichy, J. Kuneš, Phys. Rev. B 97, 235157 (2018). 